\title{
Hybrid Energy-Efficient Transmission Protocol for Heterogeneous Wireless Sensor Networks
}

\author{
Ramaswami Jothi Kavitha1, Britto Elizabeth Caroline ${ }^{2}$ \\ ${ }^{1}$ Department of ECE, University College of Engineering, Panruti (A Constituent College of Anna University), \\ Panruti, India \\ ${ }^{2}$ Department of ECE, IFET College of Engineering, Villupuram, India \\ Email: rjkeceauttpc@gmail.com, becaroline05@yahoo.com
}

Received 24 March 2016; accepted 13 May 2016; published 18 May 2016

Copyright (C) 2016 by authors and Scientific Research Publishing Inc.

This work is licensed under the Creative Commons Attribution International License (CC BY).

http://creativecommons.org/licenses/by/4.0/

(c) $\underset{\mathrm{EY}}{\mathrm{E}}$ Open Access

\begin{abstract}
Heterogeneous Wireless Sensor Networks (WSNs) have different sensing ranges and computing power in the midst of various resource constraints such as limited battery power, reduced transmit power and network potential. This paper proposes new hybrid energy efficient transmission protocol called Hybrid Transmission Protocol (HTP). The proposed algorithm focuses on the issues of throughput dreadful conditions and limited power on mobile nodes due to conflicts in multi-cell wireless networks. The design principle of the proposed routing algorithm is to introduce a new border cluster between the sink nodes and the cluster-heads in order to lengthen the lifetime of the network nodes with minimized energy consumption to attain energy efficiency. The creation of clusters is done by making use of the sensor nodes formation that has a Cluster-Head (CH) used for performing the data aggregation in the sensor nodes of the cluster. Later the data that is aggregated is transmitted with multiple hops to the base station and in turn, this process leads to the reduction in the bandwidth through the elimination of the redundant data present in a cluster. The performance results indicate that the HTP proposed gained greater network lifetime, better performance and higher throughput compared to the other available algorithms.
\end{abstract}

\section{Keywords}

Heterogeneous WSN, Border Cluster, FND, PNA

\section{Introduction}

Wireless Sensor Networks (WSNs) have been widely applied in multiple realistic domains due to its diversified 
potential [1]. The organization of sensor infrastructure is with the computing, sensing and the communication elements.

In wireless sensor networks, one among the chief restraints is the reduced amount of battery power that has a huge influence over the lifetime and also the quality of the network. Many routing protocols have been developed for wireless sensor networks in order to meet the requirement of energy utilization and resourcefulness. Scalability, Efficiency and the lifetime of a wireless sensor network can be improved by employing hierarchical routing. As observed here, sensors organize themselves into clusters and every cluster comprises of a cluster head [2]. The important role of cluster head is providing data communication between the sensor nodes and the base station in an efficient manner [3]. Another means to extend the lifetime of the wireless sensor network involves inserting a part of heterogeneous nodes. Heterogeneous wireless sensor network contains the sensor nodes having different capabilities, like diverse computing power and range of sensing. Heterogeneous wireless sensor networks prove to be very helpful in practical deployments due to proximity to situations of real life [4].

A heterogeneous wireless network is a system that provides multi-level connectivity, different levels of computing capability and energy constraints. The integration of heterogeneous wireless networks with sensor network results in heterogeneous wireless sensor network, a heterogeneous wireless sensor network is established. A heterogeneous WSN is an autonomous distributed network which sends the data via the network to the main location. Certain bottlenecks such as limited battery life, throughput capacity and contention in wireless nodes are encountered in a heterogeneous wireless sensor networks. Often, the HWSN needs to meet the network requirements. Since, the modern networks are bi- directional, enabling control over the sensor activities can be achieved. Heterogeneous wireless sensor networks provide higher bandwidth centered with cluster heads to have wider coverage and to tackle the heavy traffic in the network. The data transfer to the wired network comes into existence, only after the cluster head collects the information about the traffic. HWSN network can withstand in the complicated network environment. Energy is the rarest resource of WSN nodes, which deals with the lifetime of the network. The radio transmitter and receiver are switched off to conserve the power. HWSN needs to address the lifespan of the network, fault tolerance, self-configuration and robustness.

When compared with the homogeneous WSNs, the newly proposed HWSN routing protocols have attempted to prolong the network lifetime and period of stability, and also accomplish a reliable data transmission through the deployment of the sensor nodes having diverse capabilities. But, the proposed protocols, at times, cannot have a balance over the energy consumption of sensor nodes with efficiency. It is essential for the minimization and balance of the energy consumption among the sensor nodes present in a network in order to enhance its performance. In this paper, at first, the HTP routing algorithm is proposed and then a new border cluster is introduced between the cluster-heads and thereafter, the sink in the network will reduce the energy consumption for the purpose of extending the network lifetime and thereby attain energy efficiency. The energy consumption Pattern possesses a clear-cut meaning in [5]. To certify for an extended network lifetime, the choice of clusterheads is vital since the direction of the election process may lead to an extreme amount of energy consumption, thus resulting to the fatality of nodes specifically when a node goes very low in energy. In this way, the proposed HTP protocol is planned and executed well in order to enhance the network lifetime by guaranteeing that the cluster-heads desired are selected at each round thereby leading to a more balanced sort of energy consumption in the network.

The major contributions are listed below:

1. HTP has effectively extended to be a stable region in relation to the network lifetime by being aware of heterogeneity and transmitting cluster-head role to more proficient nodes.

2. HTP is able to achieve a stable cluster-heads by electing the network nodes, leading to an enhanced throughput in the network [6].

3. HTP is able to exploit and better distribute the added energy introduced in the network by judiciously consuming the excess energy to enhance network lifetime better than the other protocols we compared it with [5] [7].

4. HTP offers a guaranteed performance and provides an ideal solution with a uniform energy spread across the network. Hence, the nodes may die roughly at the same time.

The rest of the paper is organized as follows: Section 2 presents the significant works done and related to transmission routing schemes, followed by the description of the proposed approach and its details in Section 3. The simulation environment and the simulation results interpretation are presented in Sections 4 and the paper's conclusion is in Section 5. 


\section{Related Work}

The performance and Quality of Service is determined by the throughput of a wireless sensor networks, plays an important role in the field of research. The main cause of degradation in wireless sensor networks is the collision between the packets while transmitting the data across the network. Various scheduling algorithm has been designed to meet the QoS requirements, to reduce the energy consumption while communication and to improve the throughput.

The source of throughput degradation in a wireless sensor networks has been inspected in detail [8], based on the calculation of maximum theoretical bandwidth from the MAC scheme, modulation scheme, data rate and MSDU size. Achieving the maximum bandwidth in the network is difficult, as the heterogeneous WSN has multiple nodes, which causes interaction and interference among the nodes. As the contention node increase, the throughput falls gradually with increased back off time due to increase in the contention nodes [9]. In cause of 802.11 MAC, the throughput degradation falls even worse [10] [11] in the real time networks in accordance with the channel quality, which leads to the severe degradation in performance of the networks.

To solve the degradation problem in the networks, many algorithms has been formulated. Multiple back-to-back data packets are transmitted with the good channel quality using OAR protocol. The information fields in RTS/CTS packets are changed to enable the OAR protocol to send multiple data packets in the reserved time slot. The existing DCF [12] mechanisms and packet headers are modified by enabling this type of algorithms. Therefore, it is highly complicated to use the algorithm in the network devices.

Overlay MAC has been implemented over off-the-shell 802.11 MAC layer and needs the assumption to be provided based on MAC interface. It means scheduling over the MAC layer, using legacy network devices. Overlay MAC layer uses idle-timer mechanisms, to detect the time slots which are not used by other nodes. The network interface must be turned on, in all the network nodes-which complicate to achieve energy saving techniques. TCP and UDP [13] uses SWAN rate control mechanisms which effects on best-effort MAC [14], it doesn't requires any support for quality of service.

SWAN provides sender base admission control and service differentiation. The IP layer and the MAC layer reside between the rates controllers. The admission controller works on IP layer. Distributed mechanisms are the strength of the SWAN's model which works based on the feedback from the network. The feedback information are collected from the MAC layer or the from the other network nodes.

Scheduling and power control is combined in the work [15], in which it has cross layer framework in two phases. Initially, to eliminate the interference, the scheduling algorithm coordinates the transmission nodes in the network. Secondly, to satisfy the noise constraints the scheduled nodes controls the radio power. The algorithm works on the distributed power control, while scheduling wireless nodes. The issue with the algorithm is that it requires separate contention-free feedback channel to send information about the radio conditions. The algorithm works on central controller node, thus it is not fully distributed. The work, determines the use of only one radio channel, and scheduled by running the algorithm itself.

EEHC [16] improves the performance by introducing heterogeneity and through the election of the cluster heads on the basis of few parameters such as the average distance between a cluster head and its member nodes, average distance between the cluster head and the base station in addition to the amplifier's transmission range.

SCHS [17] described that the energy consumption during intra cluster communication is dependent on the positioning of the cluster heads in the cluster. Therefore, this scheme has partitioned the network area into two part which are the border area and inner area. Only the nodes present in the internal area have the eligibility of becoming the cluster heads and the intra cluster communication distance is reduced in this approach; thus prolonging the network lifetime. But the nodes that are residing in the border area, even with greater energy values, never get a chance of becoming a cluster head leading to an uneven distribution of energy inside a cluster.

In the work proposed, the above challenges of power management and network life time are resolved by making use of an effective hybrid transmission protocol.

\section{Proposed Method}

This work proposes a hybrid transmission protocol for the heterogeneous environment. When a multi hop is in progress, the breakdown of one relay node leads to the detachment of other nodes from the BS. The cluster heads D in the network and energy level E is considered as the key factor to achieve self adaption and energy efficiency in a sensor networks.

In order to achieve Signal-to-Noise Ratio (SNR) transmits L-bit message over a distance d and the energy 
$E_{T x}$ distributed via the radio is given as:

$$
E_{T x}(L, d)=\left\{\begin{array}{l}
L * E_{\text {elec }}+L * \varepsilon f_{s} * d^{2}, d<d_{0} \\
L * E_{\text {elec }}+L * \varepsilon_{m p} * d^{4}, d \geq d_{0}
\end{array}\right.
$$

where,

- $E_{\text {elec }} \rightarrow$ energy dissolute per bit which operates the sender (transmitter) and the receiver circuit;

- $\varepsilon f_{s}$ and $\varepsilon_{m p}$ depend on transmitter amplifier model;

- $d$ is the distance between the sender and the receiver.

An amount of energy $E_{R x}$ expends while receiving L-bit message is given as below:

$$
E_{R x}(L)=L * E_{\text {elec }}
$$

In HTP, to estimate the distance- the node joins the adjacent cluster-head $i$, on node by node basis, while constructing the cluster phase. The proposed algorithm thus provides better scalability, being less centralized whereas the existing methods require wide-ranging state information during cluster construction phase. The wireless sensor network is afforded with base station (BS) and " $n$ " sensors to support a two tier model. Assume the initial node energy in a network is known, and a pseudo code is described for initial construction of the network is given below (see algorithm) and the flow of proposed algorithm is shown in Figure 1.

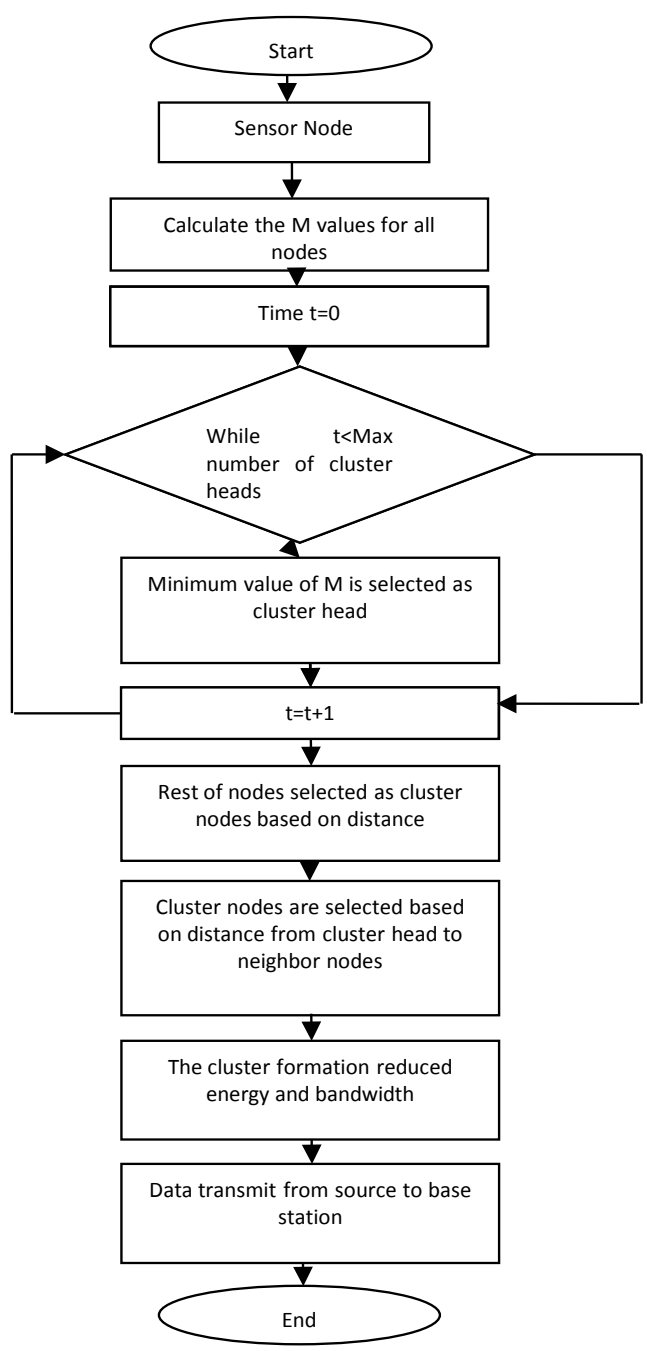

Figure 1. FND per no. of cluster-heads for DCE. 


\section{Algorithm}

1: Generate a set $\mathrm{U}(1,2, . ., \mathrm{n})$ of cluster-heads in a hierarchy

2: Compute function $\mathrm{M}=k_{1} \times \mathrm{E}+k_{2} \times \mathrm{D}$. $\mathrm{E} \rightarrow$ residual energy of a node,

$\mathrm{D}$ : the distance between a node (outside the network) and cluster-heads in the network.

3: Reorder all nodes according to their value of $\mathrm{M}$ in ascending order

4: $\mathrm{t}=0$

5: while ( $\mathrm{t}<$ Max number of cluster-heads)

6: Select a node with the minimum value of $\mathrm{M}$ as a cluster-head, and save the result in $\mathrm{U}$

$7: \mathrm{t}=\mathrm{t}+1$

8: end while

9: The rest of nodes in the network will be allocated to the closest clusters based on measuring transmission distance between them and the cluster-heads

10: Compute distance $=\mid \mathrm{U}(1,2, . ., \mathrm{n})$-the base station $\mid$

11: U.d $\leftarrow$ distance

12: Sort [U.d] in ascending order

13: Set $\mathrm{V}(1,2, . ., \mathrm{n}) \leftarrow \varnothing, \mathrm{V}$ is a set of cluster-heads in the network

14: $\mathrm{V}(1) \leftarrow \mathrm{U}(\mathrm{dmin} 1), \mathrm{V}(2) \leftarrow \mathrm{U}(\mathrm{dmin} 2), \mathrm{V}(3) \leftarrow \mathrm{U}(\mathrm{dmin} 3)$

15: $\mathrm{U}(1,2, . ., \mathrm{n}) \leftarrow \mathrm{U}(1,2, . ., \mathrm{n})-\mathrm{U}(\mathrm{dmin} 1)-\mathrm{U}(\mathrm{dmin} 2)-\mathrm{U}(\mathrm{dmin} 3)$

16: while $(\mathrm{U} /=\varnothing)$

17: For every node $i(i \in U)$, compute the distance between $i$ and each node in set $V$, save the value of the distance as a distance attribute of $\mathrm{i}$

18: $\mathrm{V}(4) \leftarrow \mathrm{U}(\mathrm{dmin} 1), \mathrm{V}(5) \leftarrow \mathrm{U}(\mathrm{dmin} 2)$,

19: $\mathrm{U} \leftarrow \mathrm{U}-\mathrm{U}(\mathrm{dmin} 1)-\mathrm{U}(\mathrm{dmin} 2)$

20: For every node i in set $U$, compute the distance between $i$ and each nodein set $V$, save the value of the distance as i.d

$$
\begin{aligned}
& \text { 21: } \mathrm{V}(6) \leftarrow \mathrm{U}(\mathrm{dmin} 1), \mathrm{V}(7) \leftarrow \mathrm{U}(\mathrm{dmin} 2) \\
& \text { 22: } \mathrm{U} \leftarrow \mathrm{U}-\mathrm{U}(\mathrm{dmin} 1)-\mathrm{U}(\mathrm{dmin} 2) \\
& \text { 23: ... } \\
& \text { 24: end while } \\
& \text { 25: Output } \mathrm{V}
\end{aligned}
$$

The sensor network has three tiers namely sensor tier, a relay tier and a BS tier. Here, most of the belongs to sensor tier, and are hierarchical. The node bypasses connection across the sensor tier and helps to communicate with each other in a relay tier.

Initially, while constructing the network structure, the routing problem busted into two different phases:

1) with the global information the cluster heads are resolute and the architecture is initiated in the global planning phase

2) on a node by node basis, the cluster joining nodes are shaped with local phase. In the latter, senor nodes are assembled into cluster.

For the purpose of collecting sensor data, HTP utilizes cluster head $(\mathrm{CH})$ which has information of sensor states and multi hop routing set up.

The cluster head is designated based on its location in the sensor network and its energy reservoir perception. By the hierarchical construction of the three tier model, clusters are merged and formed. For constructing the initial network, the metric value $\mathrm{M}$ along with the potential of the cluster head should be known for calculation.

$$
M=K_{1} \times E+K_{2} \times D
$$

where,

- The size is normalized so that $E \in(0,1), D \in(0,1), k_{1} \in(0,1)$ and $k_{2} \in(0,1)$,

- $E \rightarrow$ residual energy of a node,

- $D \rightarrow$ distance between a node (outside the network) and cluster-heads in the network,

- $k_{1} \& k_{2} \rightarrow$ weights.

The base station broadcasts the control messages from the sink node to the source node. The sink node receives the energy metric E of nodes in the network operation at the initial round. The HTP control packet sends control messages to both the network nodes and the base station. The base station saves all the possible routes of 
the network in the routing table. By multi-hop routing, the base station broadcasts the control messages to the cluster heads and the normal nodes. HTP messages are transmitted from cluster heads to the base station via multi hop routes. The base station decides the routes to the nodes only after receiving the notification of the cluster head death, and rebuilt the route correspondingly.

The base station sends control message to the normal nodes and the cluster heads, to maintain the existing network for each time slots to verify whether the nodes are still alive. The energy level information and the routing table of the base station will be updated based on the received information. The cluster head and the sources are broadcasted with the updated information. The dead cluster member is deleted when the cluster head receives the control message. With response to the partitioning and the node failure, group reorganization may be performed.

Communication may be interrupted with the failure in the Tier 1of the network. The optimal path is chunked, with the dynamic load imbalance in the sensor nodes due to the failure in the network nodes. The dead cluster head may be replaced by triggering the mechanism to select a new cluster-head for the complete network, thereby restoring the communication interrupt.

\section{Results and Discussion}

This section discusses and evaluates the experimental results of the new Hybrid Transmission Protocol (HTP). Then the HTP performance is compared with the already available Low Energy Adaptive Clustering Hierarchy (LEACH) [18] protocol. For the purpose of the evaluation of the performance, this work employed the NS2 simulator. Specifications of the Simulations utilized in this work are tabulated in Table 1 as below. A $100 \mathrm{~m} \times$ $100 \mathrm{~m}$ of randomly distributed heterogeneous nodes with their initial energies varying between $0.5 \mathrm{~J}$ to $2.25 \mathrm{~J}$ is used and BS is positioned in the center of the network system. In order to be fair, the energy of the system on an overall for every protocol is assured to be the same; a total energy of $102.5 \mathrm{~J}$ has been used. In addition, the optimal parameters of these protocols are used for yielding their corresponding best performance. In the case of LEACH and HTP, $20 \%$ of the nodes are provided with each of $2 \mathrm{~J}$ of energy and $50 \%$ with $0.5 \mathrm{~J}$ of energy.

\subsection{Performance Evaluation}

This work considered the following important Performance metrics for the evaluation by simulation

\subsubsection{FND (Stability Period Is Also Known as First Node Dies)}

The number of cluster-heads varies from 1 and 30. This Figure 2 reveals that as the cluster-heads increases from 0 to 10, the FND improves significantly. The HTP curve is in between 10 to 20 cluster-heads with a point inbetween, ahead of 20 cluster-heads, the curve starts downwards. The optimality of Nopt lies around 16 clusterheads for our setup. The non-cluster head nodes will often transmit data to the cluster heads of large distances, when there is only few cluster heads; this drains their energy faster. The non-cluster head nodes can easily trace a nearby cluster-head, when the cluster head ranges from 10 to 20. There is not much data aggregation being performed, when the number of CHs ranges beyond 20. More energy will be expended due to the smaller sizes of the clusters formed. HTP is stable, when the number of cluster-head ranges from 10 to 20 . This shows the robustness of HTP. The variable number of cluster-head election, degrades the energy consumption elegantly [10] [14].

Table 1. Table parameter settings.

\begin{tabular}{cc}
\hline Parameter & Value \\
\hline Eelec & $50 \mathrm{~nJ} / \mathrm{bit}$ \\
EDA & $5 \mathrm{~nJ} / \mathrm{bit} / \mathrm{message}$ \\
Eo & $0.5 \mathrm{~J}$ \\
$\mathrm{k}$ & 4000 \\
Popt & 0.1 \\
Efs & $10 \mathrm{pJ} / \mathrm{bit} / \mathrm{m} 2$ \\
Emp & $0.0013 \mathrm{pJ} / \mathrm{bit} / \mathrm{m} 4$ \\
$\mathrm{n}$ & 100 \\
\hline
\end{tabular}




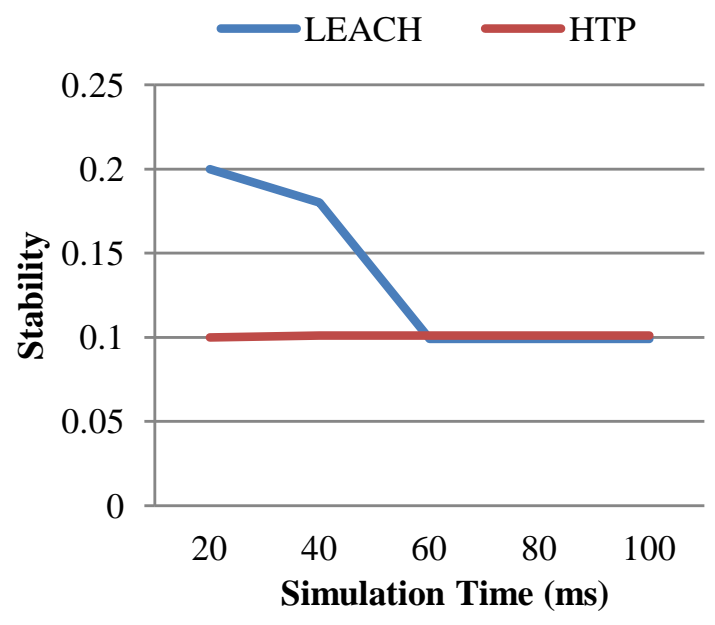

Figure 2. FND per no. of cluster-heads for DCE.

\subsubsection{PNA (Percent Nodes Alive, 90)}

Figure 3 shows the life span of the HTP and LEACH. The LEACH protocol is designed to attain robustness in a homogeneous setup; the HTP is tested for attaining performance based on these criteria. Based on the simulation procedure, HTP yields better performance compared to the existing method, which is also demonstrated in Figure 3. The LEACH performance is also acceptable, for a critical application that needs about $80 \%-100 \%$ full monitoring requirements, HTP provides evidence to be more suitable. This is quite an important result for the HTP protocol and it is one of the key differences between the HTP and the other protocols.

\subsubsection{Energy Consumption}

Figure 4 shows the Variation in energy consumption pattern, when there is a change in the number of clusters "c" formed. Energy values in log-scale are the unit of Joules [19] [20]. Figure 4 furnishes, the average energy devoted by each individual of the non- $\mathrm{CH}$ sensor node and $\mathrm{CH}$ sensor node, and the energy put away (total) in each round is plotted against by varying the no. of clusters (c) for a typical clustered network setting. While the total energy strikes the bottom with copt $=10$, it is insensitive to the cluster as shown on the log-scale plot [5]. It is clearly shown that for each node the energy consumption of the $\mathrm{CH}$ is much more important than a non- $\mathrm{CH}$ node, about an order of magnitude more over a wide range of cluster values.

\subsubsection{Throughput}

Throughput is defined as the total number of messages that are delivered over the total simulation time. In mathematical terms, it can be defined as:

$$
T p=N / 1000
$$

where $N$ refers to the number of bits that are received with success by every destination.

Figure 5 illustrates the results of the comparison of throughput made between the proposed HTP technique and the available LEACH technique. It is observed that the proposed HTTS achieves higher throughput in comparison with LEACH.

End-to-End Delay (EED): The end-to-end delay is computed by getting the time delay suffered by a message when transmitted from the source nodes to the destination node.

$$
\mathrm{EED}=\sum(\text { Messege Reach Time }- \text { Messegesend Time }) / \sum \text { Number of Connections }
$$

Figure 6 shows the comparison of the end-to-end delay between the proposed HTP with the already available LEACH technique. In case the size is increased, the HTP employs an active energy consumption strategy for detecting the possible link breaks between the source and destination nodes and thereafter quickly applies an alternate path finding mechanism for the purpose of message transfer. Furthermore, when the number of nodes is increased, the proposed technique establishes a decrease in delay rather than seen in the other available techniques. 


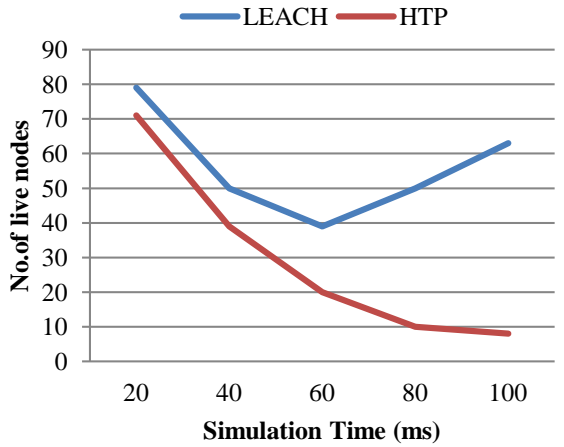

Figure 3. Live nodes/round.

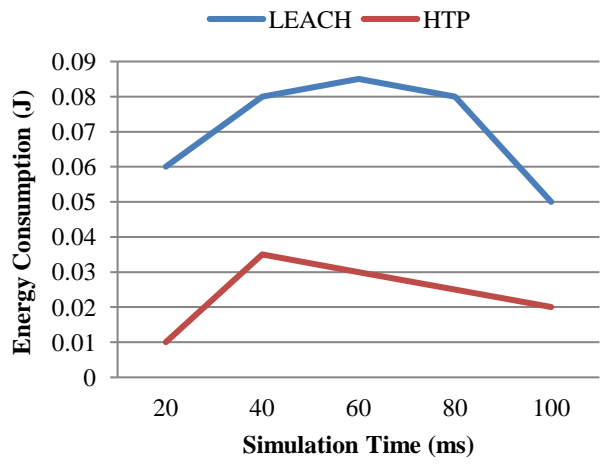

Figure 4. The variation in energy consumption pattern.

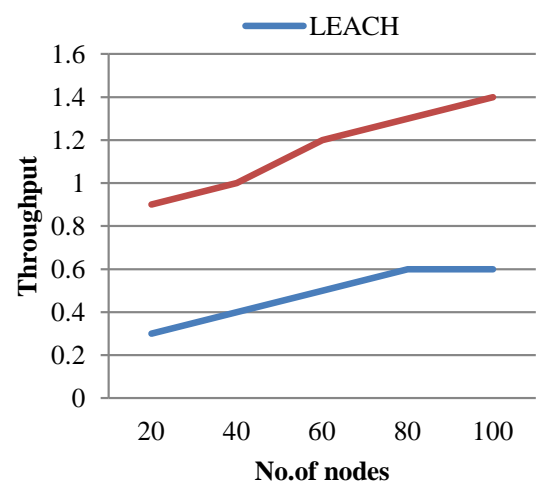

Figure 5. Throughput vs. no of nodes.

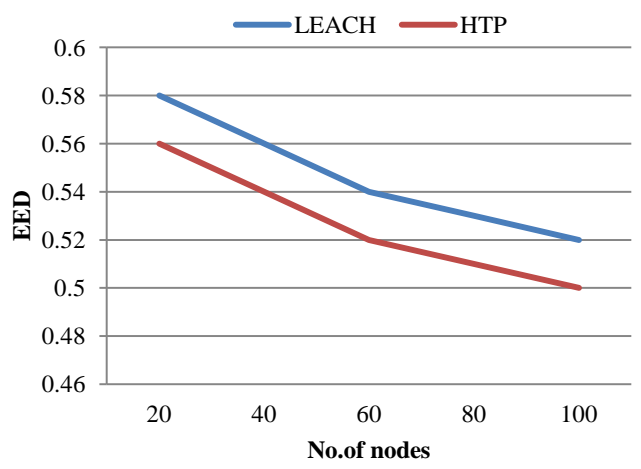

Figure 6. EED vs. no of nodes. 


\section{Conclusion}

Limiting energy consumption is one of the most pressing problems for many real-world deployments of wireless sensor networks. As sensor nodes are battery-powered, energy optimization has been one of the main objectives for a robust protocol design. In this research work, a novel Hybrid Transmission Protocol (HTP) is introduced for heterogeneous wireless sensor networks. This cluster proposed consists of a Cluster-Head (CH) which carries out data aggregation of the sensor nodes and thereafter transmits them to the base station by multiple hops and minimizes the bandwidth through the elimination of the redundant data present in a cluster. Thus, the transmission protocol improves the energy-efficiency in the case of a deployment that is hierarchically clustered. The experimental outcomes exhibit that the new HTP achieves reduced end to end delay and higher throughput in comparison with the existing LEACH protocol. Future work will chiefly concentrate on the techniques for further balancing the energy consumption of each node by making use of the unequal clusters and over the mobile heterogeneous sensor nodes. Also, the whole energy issue is to be resolved in the network.

\section{References}

[1] Villas, L., Boukerche, A., Ramos, H.S., et al. (2013) DRINA: A Lightweight and Reliable Routing Approach for InNetwork Aggregation in Wireless Sensor Networks. IEEE Transactions on Computers, 62, 676-689. http://dx.doi.org/10.1109/TC.2012.31

[2] Rashed, Md.G., Kabir, M.H. and Ullah, S.E. (2011) WEP: An Energy Efficient Protocol for Cluster Based Heterogeneous Wireless Sensor Network. International Journal of Distributed and Parallel Systems (IJDPS), 2, 54-60. http://dx.doi.org/10.5121/ijdps.2011.2205

[3] Saini, P. and Sharma, A.K. (2010) Energy Efficient Scheme for Clustering Protocol Prolonging the Lifetime of Heterogeneous Wireless Sensor Networks. International Journal of Computer Applications, 6, 30-36.

[4] Kumar, D., Aseri, T.C. and Patel, R.B. (2010) Distributed Cluster Head Election (DCHE) Scheme for Improving Lifetime of Heterogeneous Sensor Networks. Tamkang Journal of Science and Engineering, 13, 337-348.

[5] Demers, A. (1989) Analysis and Simulation of a Fair Queuing Algorithm. In ACM SIGCOMM Computer Communication Review, 19, 1-12. http://dx.doi.org/10.1145/75247.75248

[6] Waharte, S., Xiao, J. and Boutaba, R. (2004) Overlay Wireless Sensor Networks for Application-Adaptive Scheduling in WLAN. In: Mammeri, Z. and Lorenz, P., Eds., High Speed Networks and Multimedia Communications, Springer, Berlin Heidelberg, 676-684. http://dx.doi.org/10.1007/978-3-540-25969-5 61

[7] Hohlt, B., Doherty, L. and Brewer, E. (2004) Flexible Power Scheduling for sensor Networks. Proceedings of the 3rd International Symposium on Information Processing in Sensor Networks, Berkeley, 26-27 April 2004 205-214. http://dx.doi.org/10.1145/984622.984653

[8] Jun, J., Peddabachagari, P. and Sichitiu, M. (2003) Theoretical Maximum Throughput of IEEE 802.11 and Its Applications. IEEE International Symposium on Network Computing and Applications, Cambridge, 18 April 2003, 249-256.

[9] Heusse, M., Rousseau, F., Berger-Sabbatel, G. and Duda, A. (2003) Performance Anomaly of 802.11b. 22nd Annual Joint Conferences of the IEEE Computer and Communications, San Francisco, 30 March- 3 April 2003, 836-843.

[10] Choi, S., Park, K. and Kim, C. (2005) On the Performance Characteristics of WLANs: Revisited. ACM SIGMETRICS Performance Evaluation Review, 33, 97-108. http://dx.doi.org/10.1145/1071690.1064225

[11] Vasan, A. and Shankar, A.U. (2002) An Empirical Characterization of Instantaneous Throughput in 802.11b WLANs. Technical Report, Department of Computer Science, University of Maryland, College Park.

[12] Wu, X.C. and Ananda, A.L. (2004) Link Characteristics Estimation for IEEE 802.11 DCF Based LAN. In 29th Annual IEEE International Conference on Local Computer Networks, Tampa, 16-18 November 2004, 302-309. http://dx.doi.org/10.1109/LCN.2004.73

[13] Wu, X.C. (2004) Simulate 802.11b Channel within ns2. Technical Report, School of Computing, National University of Singapore, Singapore.

[14] Cidon, I. and Sidi, M. (1989) Distributed Assignment Algorithms for Multihop Packet Radio Networks. IEEE Transactions on Computers, 38, 1353-1361. http://dx.doi.org/10.1109/12.35830

[15] Elbatt, T. and Ephremides, A. (2004) Joint Scheduling and Power Control for Wireless Ad Hoc Networks. IEEE Transactions on Wireless communications, 3, 74-85. http://dx.doi.org/10.1109/TWC.2003.819032

[16] Kumar, D., Patel, R.B. and Aseri, T.C. (2009) EEHC: Energy Efficient Heterogeneous Clustered Scheme for Wireless Sensor Network. Computer Communications, 32, 662-667. http://dx.doi.org/10.1016/j.comcom.2008.11.025

[17] Pal, V., Singh, G. and Yadav, R.P. (2012) SCHS: Smart Cluster Head Selection Scheme for Clustering Algorithms in 
Wireless Sensor Networks. Wireless Sensor Network, 4, 273-280.

[18] Sujee, R. and Kannammal, K.E. (2015) Behavior of LEACH protocol in Heterogeneous and Homogeneous Environment. International Conference on Computer Communication and Informatics (ICCCI), Coimbatore, 8-10 January 2015, 1-8. http://dx.doi.org/10.1109/ICCCI.2015.7218126

[19] Snow, J., Feng, W. and Feng, W. (2005) Implementing a Low Power TDMA Protocol over 802.11. IEEE Wireless Communications and Networking Conference, Vol. 1, 75-80. http://dx.doi.org/10.1109/wcnc.2005.1424479

[20] Jamieson, K. (2002) Implementation of a Power-Saving Protocol for Ad Hoc Wireless networks. Master's Thesis, Massachusetts Institute of Technology, Cambridge. 